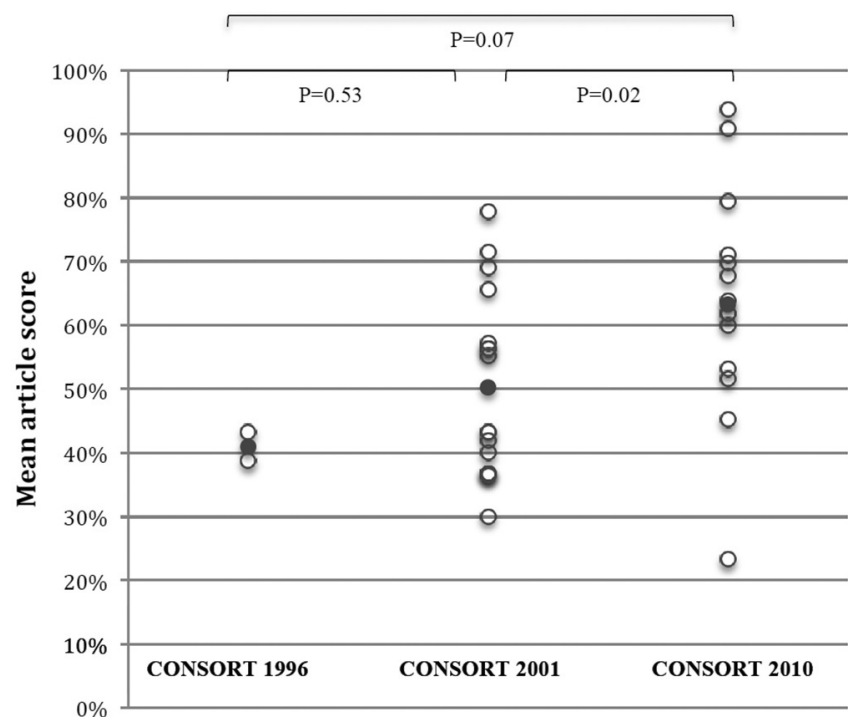

Abstract 3 Figure 2 Individual study CONSORT 2010 score (open) grouped by available CONSORT statement $(1996,2001$ or 2010) at time of study publication. Mean score (filled) shows increasing mean scores with revisions to CONSORT statement. Increase after introduction of CONSORT 2010 was significant (difference $+13.1 \%$, $P=0.02$ )

$31 / 32$ ) and result interpretation (22) $(96.9 \%, 31 / 32)$. The worst reported were reporting of binary outcomes $(17 b)(0 \%$, 0/7), abstract $(1 b)(9.4 \%, 3 / 32)$, allocation concealment mechanism (9) $(12.5 \%, 4 / 32)$ and trial ending (14b) $(12.5 \%, 4 / 32)$ (Figure 1). The reporting scores showed highly significant correlation with journal impact factor $(\mathrm{r}=0.54, \mathrm{P}<0.01), 5$ year impact factor $(\mathrm{r}=0.49, \mathrm{P}<0.01)$ and publication year $(\mathrm{r}=0.49, \mathrm{P}<0.01)$. Mean scores increased after publication of each updated CONSORT statement: from $41.0 \%$ (range $38.7-43.3 \%$, SD 3.3$)$ to $48.6 \%(30.0-77.8 \%$, SD 14.5) after CONSORT $2001(p=0.53)$, and from $48.6 \%$ to $63.3 \%$ $(23.3-93.8 \%$ SD 17.6) after CONSORT $2010(\mathrm{P}=0.02)$ (Figure 2).

Conclusion This study identified all RCTs involving pharmacological interventions in HFPEF, and demonstrated that while reporting standards have improved with time, at present the majority of studies do not meet the CONSORT standards for reporting of RCTs. Better compliance is needed.

\section{$4 \quad$ MORTALITY IS HIGHER IN HEART FAILURE PATIENTS DISCHARGED FROM HOSPITAL ON WEEKENDS}

${ }^{1}$ Paul Carter*, ${ }^{2}$ Hardeep Uppal, ${ }^{3}$ Suresh Chandran, ${ }^{4}$ Jaydeep Sarma, ${ }^{2}$ Rahul Potluri. ${ }^{1}$ The Royal Free London NHS Foundation Trust; ${ }^{2}$ ACALM Study Unit in Collaboration with Aston Medical School; ${ }^{3}$ Department of Acute Medicine, North Western Deanery; ${ }^{4}$ University Hospital South Manchester; *Presenting Author

\subsection{6/heartjnl-2016-309890.4}

Introduction Heart failure is common and one of the top contributors to mortality in the United Kingdom. There is some highly controversial evidence to support a "weekend-effect" with mortality rates elevated for patients admitted or discharged from hospital on the weekend. The impact of weekend discharge on mortality rates of heart failure patients in the United Kingdom has not been previously studied though, and was the focus of this study. We did this using a large database of patients discharged with heart failure to hospitals in the North of England, UK between 2000-2013.

Methods Anonymous information on patients with heart failure was obtained from hospitals in North England, UK between $1^{\text {st }}$ January 2000 and $31^{\text {st }}$ March 2013. This data was analysed according to the ACALM (Algorithm for Comorbidities, Associations, Length of stay and Mortality) study protocol. ICD-10 and OPCS-4 codes were used to trace patients coded for heart failure, day of discharge, patient demographics, prevalence of comorbidites and mortality data. The impact of day of discharge on mortality in heart failure patients was analysed by Kaplan-meier survival analysis and cox regression analysis adjusted for age, gender, ethnicity and the 9 top contributors to mortality (Ischaemic Heart Disease, colon cancer, breast cancer, lung cancer, pneumonia, cerebrovascular disease, Chonic Obstructive Pulmonary Disease and dementia). $\mathrm{P}$ values of $<0.05$ were taken as statistically significant.

Results Of 929552 patients admitted during the study period there were 31760 patients with heart failure. 27944 (88.8\%) of patients were discharged on weekdays and 3816 (11.2\%) were discharged on weekends. Demographics for patients discharged on weekdays (mean age $74 \pm 14$ and $50.3 \%$ male) was similar to those discharged on weekends $(74 \pm 14$ and $50.1 \%$ male). Similarly, prevalence of the 9 top contributing conditions to mortality in the UK were similar in both groups (see Table 1). Crude all-cause mortality for heart failure patients discharged on weekends (69.2\%) was higher than those discharged on weekdays (66.0\%). Mortality was

Abstract 4 Table 1 Demographics of heart failure patients discharged on weekdays and weekends

\begin{tabular}{|l|c|c|}
\hline & Discharged on Weekday & Discharged on Weekend \\
\hline $\mathbf{N}$ & 27944 & 3816 \\
\hline Mean agetsd (years) & $74 \pm 14$ & $74 \pm 14$ \\
\hline Male, $\mathbf{n} \%$ & $14054(50.3 \%)$ & $1911(50.1 \%)$ \\
\hline$\%$ dead, $\mathbf{n} \%$ & $18450 / 27944(66.0 \%)$ & $2640 / 3816(69.2 \%)$ \\
\hline $\begin{array}{l}\text { Mean Length of } \\
\text { Hospital stay } \mathbf{s d} \text { (days) }\end{array}$ & $11.8 \pm 21.2$ & $10.1 \pm 17.2$ \\
\hline
\end{tabular}


Abstract 4 Table 2 Prevalence of top contributors to mortality amongst heart failure patients discharged on weekdays and weekends

\begin{tabular}{|l|c|c|}
\hline & $\begin{array}{c}\text { Discharged on } \\
\text { Weekday }\end{array}$ & Discharged on Weekend \\
\hline Heart Failure & $27944(100 \%)$ & $3816(100 \%)$ \\
\hline IHD & $12052(43.1 \%)$ & $1592(41.7 \%)$ \\
\hline Lung Cancer & $505(1.8 \%)$ & $63(1.7 \%)$ \\
\hline Breast Cancer & $543(1.9 \%)$ & $90(2.4 \%)$ \\
\hline Colon Cancer & $198(0.7 \%)$ & $38(1.0 \%)$ \\
\hline $\begin{array}{l}\text { Cerebrovascular } \\
\text { Disease }\end{array}$ & $2344(8.4 \%)$ & $311(8.1 \%)$ \\
\hline COPD & $5699(20.4 \%)$ & $690(18.1 \%)$ \\
\hline CKD & $4338(15.5 \%)$ & $543(14.2 \%)$ \\
\hline Dementia & $1894(6.8 \%)$ & $258(6.8 \%)$ \\
\hline Pneumonia & $4490(16.1 \%)$ & $619(16.2 \%)$ \\
\hline
\end{tabular}

statistically greater for heart failure patients discharged on weekends after cox regression analysis accounting for differences in age, gender, ethnicity and the top contributors to mortality (OR 1.270, 95\% confidence intervals 1.219-1.323). Kaplan-Meier survival analysis demonstrated that although 5year survival for heart failure patients discharged on MondayFriday were similar, survival for those discharged on Saturday/ Sunday was greatly reduced.

Conclusions We have demonstrated that long-term mortality of heart failure patients discharged on weekends is significantly higher than those discharged on weekdays. Further research is required to elucidate the reasons for these disparities and could relate to premature discharge or lack of community care for heart failure patients discharged on weekends.

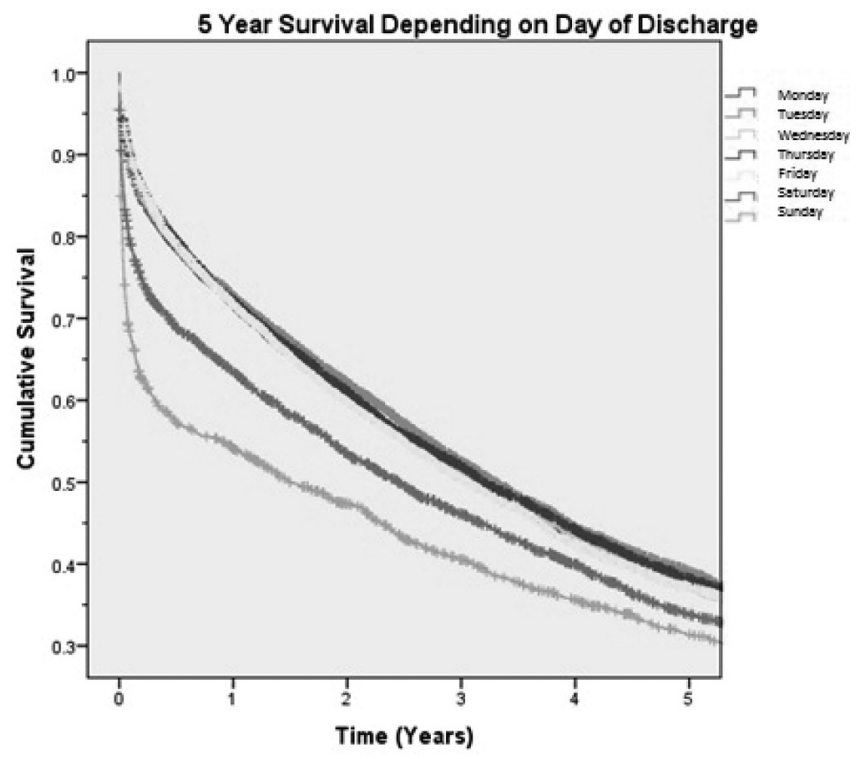

Abstract 4 Figure 15 year survival depending on day of discharge
5 THE IMPACT OF PSYCHIATRIC COMORBIDITIES ON THE LENGTH OF HOSPITAL STAY IN PATIENTS WITH HEART FAILURE

${ }^{1}$ Paul Carter*, ${ }^{2}$ Andrew Carter, ${ }^{3}$ Jennifer Reynolds, ${ }^{2}$ Hardeep Uppal, ${ }^{4}$ Suresh Chandran, ${ }^{2}$ Rahul Potluri. ' Royal Free London NHS Foundation Trust; ${ }^{2} A C A L M$ Study Unit in Collaboration with Aston Medical School; ${ }^{3}$ University of Birmingham; ${ }^{4}$ Department of Acute Medicine, North Western Deanery; *Presenting Author

\subsection{6/heartjnl-2016-309890.5}

Introduction Heart failure (HF) is a major healthcare problem contributing significantly to hospital admission stays and NHS spending. Reducing length of hospital stay (LOS) in HF is paramount in reducing this burden and is influenced by factors relating to the condition, sociodemographics and comorbidities. Psychiatric comorbidities are being increasingly identified amongst HF patients but their impact on LOS has not been studied in the UK.

Objectives We aimed to investigate the impact of psychiatric comorbidities on length of hospital stay in a large database of patients admitted with heart failure to hospitals in the North of England over a 14-year period between 2000-2013.

Methods Anonymous information on heart failure patients was obtained from the local health authority hospital activity register for hospitals in North West England between 1st January 2000 and $31^{\text {st }}$ March 2013. This data was analysed according to the ACALM (Algorithm for Comorbidities, Associations, Length of stay and Mortality) study protocol. ICD-10 and OPCS-4 codes were used to trace HF patients, psychiatric comorbidities and demographics including LOS. LOS of HF patients with and without psychiatric comorbidites were compared by logistic regression. $\mathrm{P}$ values of $<0.05$ were taken as statistically significant.

Results Of 929552 patients admitted during the study period 31760 patients had heart failure. The mean age of heart failure patients was $73.6,50.3 \%$ were male and the majority were of Caucasian origin (85.1\%). Amongst $31760 \mathrm{HF}$ 